\title{
Optimierte Polymer-Rohrwerkstoffe für effiziente Drainagesysteme in Tunnelbauwerken-PolyDrain Teil II
}

\author{
Florian Arbeiter ${ }^{1}$, Stefanie Eichinger ${ }^{2}$, Gisbert Rieß ${ }^{3}$, Tobias Schachinger ${ }^{4}$, Ronny Boch ${ }^{2,5}$, Robert Wenighofer ${ }^{6}$, \\ Robert Galler ${ }^{6}$, Andreas Hausberger ${ }^{7}$, Elmar Strobl ${ }^{8}$, Michael Stur ${ }^{9}$, Florian Saliger ${ }^{4}$, Michael Steiner ${ }^{10}$, \\ Martin Dietzel ${ }^{2}$ und Gerald Pinter ${ }^{1}$
}

\begin{abstract}
${ }^{1}$ Lehrstuhl für Werkstoffkunde und Prüfung der Kunststoffe, Montanuniversität Leoben, Leoben, Österreich ${ }^{2}$ Institut für Angewandte Geowissenschaften, Technische Universität Graz, Graz, Österreich ${ }^{3}$ Lehrstuhl für Chemie der Kunststoffe, Montanuniversität Leoben, Leoben, Österreich ${ }^{4}$ Österreichische Bundesbahnen ÖBB Infrastruktur AG, Wien, Österreich ${ }^{5}$ Geoconsult ZT GmbH, Wissenspark Salzburg Urstein, Puch bei Hallein, Österreich ${ }^{6}$ Lehrstuhl für Subsurface Engineering, Montanuniversität Leoben, Leoben, Österreich ${ }^{7}$ Polymer Competence Center Leoben GmbH, Leoben, Österreich ${ }^{8}$ Ingenieurbüro Strobl, Kumberg, Österreich Institut für Angewandte Geologie, Universität für Bodenkultur Wien, Wien, Österreich ${ }^{10}$ Autobahnen- und Schnellstraßen-Finanzierungs-Aktiengesellschaft ASFINAG, Wien, Österreich
\end{abstract}

Eingegangen 14. Oktober 2020; angenommen 19. Oktober 2020; online publiziert 6. November 2020

Zusammenfassung: Um notwendige Reinigungsarbeiten zur Aufrechterhaltung der Drainagewirkung von Drainagerohren in Tunneln zu minimieren, wurden im gegenständlichen Projekt Kunststoffe mit verschiedenen aktiven Wirkstoffen modifiziert, welche die Versinterungsneigung an den Rohroberflächen reduzieren sollen. Ausgehend von dieser praktischen Fragestellung wurden sieben verschiedene Rezepturen hinsichtlich ihres Potentials zur Verringerung der karbonatischen Versinterungen und zur industriellen Verarbeitbarkeit bewertet. Die hergestellten Compounds aus Matrix-Kunststoff, einem kommerziell verfügbaren Polyethylen-Rohr Typ und dem jeweiligen Wirkstoff sind zu Prüfkörpern verarbeitet und sowohl in Laborals auch in Feldversuchen in realen Tunnelbauwerken ausgelagert worden. Anschließend fand die Beurteilung der Probekörper hinsichtlich der aufgewachsenen Versinterungen mittels chemischem Säureaufschluss, sowie optischen Analyseverfahren statt. Hinsichtlich der Verringerung der Versinterung zeigten das Polyethylen-Glykol Copolymer sowie das Compound mit Zeolith die besten Effekte.

Dr. F. Arbeiter ( $\triangle)$

Lehrstuhl für Werkstoffkunde und Prüfung der Kunststoffe

Montanuniversität Leoben,

Franz-Josef-Str. 18,

8700 Leoben, Österreich

florian.arbeiter@unileoben.ac.at
Schlüsselwörter: Tunnelbau, Drainagerohr, Versinterung, Feldversuche, Kunststoffrohr

Optimized Polymer Pipe Materials for Efficient Drainage Systems in Tunnelling Applications-PolyDrain Part II

Abstract: Aim of this project was to develop modified polymers which can reduce the precipitation of scale deposits in tunnel drainage pipes. This is necessary to reduce the maintenance costs for cleaning and flushing of the pipes. Based on this issue, 7 different compounds, consisting of a polyethylene base polymer and active fillers were developed. Selection of the fillers was based on their potential to reduce precipitations of scale deposits as well as largescale processability. The developed compounds were processed into specimens and exposed to drainage-waters both in laboratory as well as field tests in actual tunnels. Subsequently, $\mathrm{CaCO}_{3}$ precipitations on the materials were analyzed using chemical and optical methods. Regarding the efficacy, especially the compounds with polyethyleneglycol and zeolite proved to be very promising.

Keywords: Tunneling, Drainage pipe, Scale deposits, Field experiments, Polymer pipe 


\section{Einleitung}

Aufbauend auf den Arbeiten, die im Artikel „Optimierte Polymer-Rohrwerkstoffe für effiziente Drainagesysteme in Tunnelbauwerken - PolyDrain“ [1] beschrieben wurden, sollen im gegenwärtigen Artikel die Ergebnisse hinsichtlich optimierter Polymer-Rohrwerkstoffe für den Einsatz in Drainagesystemen präsentiert werden. Das konkrete Ziel der Arbeit war es, Kunststoffe, die für zukünftige Drainagerohre in Tunnelbauwerken zum Einsatz kommen könnten, so zu modifizieren, dass es zu einer geringeren Versinterungsneigung kommt. Da die Reinigung von Drainagerohren mittels Hochdruckspülung oder anderer Verfahren sehr zeit- und kostenintensiv ist, wäre somit ein in Summe effizienterer Betrieb der auf eine lange Nutzungsdauer ausgelegten Bauwerke möglich $[2,3]$.

\section{Experimentelles}

\subsection{Materialentwicklung}

Ziel des gegenwärtigen Projekts war es, Kunststoffe mittels Füll- bzw. Wirkstoffen derart zu modifizieren, dass es zu einer geringeren Versinterungsneigung in den daraus gefertigten Drainagerohren kommt. Ausgehend von dieser Aufgabenstellung sowie dem Fachwissen aus der verfügbaren Literatur [4-8], wurden die nachfolgenden, in Tab. 1 dargestellten Formulierungen von Kunststoffen ausgewählt. Zur Herstellung der zu untersuchenden Proben wurden Compounds mittels Kneter- und Plattenpresse hergestellt. Aus den so entstandenen Platten wurden je nach Versuch die notwendigen Probekörper entnommen.

\subsection{Auslagerungsversuche}

Um die Wirksamkeit der entwickelten Compounds unter möglichst realen Bedingungen zu prüfen, wurden unterschiedliche Auslagerungsmethoden, bei denen es zu einem Aufwachsen von Versinterungen auf den Proben kommen soll, ausgewählt und durchgeführt. Um verschiedene Szenarien in Drainagerohren nachzustellen, wurden Auslagerungsorte mit verschiedenen Fließgeschwindigkeiten,
pH-Werten und Wasseraufkommen ausgewählt. Zusätzlich wurden Versuche sowohl im Labor unter kontrollierten Bedingungen als auch in realen Bauwerken durchgeführt. Eine genauere Beschreibung der Auslagerungsorte und deren Beschaffenheit ist nachfolgend aufgelistet.

\subsubsection{Dynamische Versuche}

Als dynamische Versuche werden nachfolgend alle Versuche bezeichnet, bei denen es einen kontinuierlichen Wasserfluss über die Proben gibt. Dies beinhaltet sowohl den im Folgenden dargestellten Laborversuch, als auch Auslagerungen in realen Drainagerohren und Tunnelentwässerungen.

Für die erste Versuchsreihe unter klar kontrollierbaren Randbedingungen wurde der in Abb. 1 und 2 dargestellte Versuchsaufbau in Rinnenform an der TU-Graz entwickelt [1, 3, 9]. Der Prüfstand simuliert im Labormaßstab die Abscheidung von Kalziumkarbonat-Mineralen aus einer an diesen Mineralen übersättigten Lösung. Der Fokus der Untersuchungen liegt auf der Kristallisation von Kalziumkarbonat-Mineralen an der Oberfläche individueller Kunststoffe. Die chemische Zusammensetzung, Temperatur und Fließrate der Versuchslösung sind dabei so gewählt, dass karbonatische Ausfällungen innerhalb von Minuten bis zu einigen Tagen auftreten. Hierfür wird ein Versuchswasser (Reaktionslösung) kontinuierlich über die Mischung einer beispielsweise 10 millimolaren $(\mathrm{mM}) \mathrm{CaCl}_{2}+0,1 \mathrm{mM} \mathrm{SrCl}_{2}$ Stammlösung mit einer $10 \mathrm{mM} \mathrm{NaHCO}_{3}$ haltigen Stammlösung generiert und diese gemischte Reaktionslösung über eine $2 \mathrm{~m}$ lange Fließstrecke über laminares Fließen drainiert, wobei die Durchflussgeschwindigkeit etwa $9 \mathrm{ml} / \mathrm{min}$ beträgt (siehe Abb. 1). Die Experimentdauer betrug in diesem Ansatz 10 Tage bei einer konstanten Wassertemperatur von $20^{\circ} \mathrm{C}$ und einem initialen $\mathrm{pH}-$ Wert von $8,0 \pm 0,1$. In diesem labordesignten Teststand werden die praxisrelevanten Parameter, wie pH-Wert, Lösungsinhaltstoffe und Temperatur, sowie die räumlich-zeitliche Entwicklung der Zusammensetzung des Wassers und der karbonatischen Abscheidungen an den Kunststoffoberflächen entlang der Fließstrecke in-situ und über verteilte Probennahmen und anschließende hydro- und materialchemische Analysen untersucht. Die zu testenden Kunststoffe sind hierbei jeweils am Anfang und am Ende von zwei parallel aufgebauten Fließ-

TABELLE 1

Untersuchte Materialrezepturen zur Überprüfung ihrer Wirksamkeit hinsichtlich der Versinterungsneigung

\begin{tabular}{|c|c|c|c|}
\hline Matrix & Füll- bzw. Wirkstoff & Kennung & Wirkungsmechanismus \\
\hline \multirow{8}{*}{$\begin{array}{l}\text { PE-HD } \\
\text { Rohrma- } \\
\text { terial } \\
\text { (PE100) }\end{array}$} & - & $\mathrm{PE}$ & Benchmark \\
\hline & $4 \%$ Silan $+0,5 \%$ Peroxid & PE + Silan \& Peroxid & Oberflächenpolarität \\
\hline & $4 \%$ Polyethylen-Glykol Copolymer & $\mathrm{PE}+\mathrm{PEG}$ & Oberflächenpolarität \\
\hline & $4 \%$ Natriumstearat & $\mathrm{PE}+\mathrm{Na}$-Stearat & Chem. Puffer im sauren Bereich \\
\hline & 4\% Ammonium-polyphosphat & PE +Amm.Phosphat & Chem. Puffer im sauren und basischen Milieu \\
\hline & $4 \%$ Stearinsäure & PE + Stearinsäure & Chem. Puffer im basischen Bereich \\
\hline & $4 \%$ Zeolith $^{\mathrm{a}}$ & PE + Zeolith & Ionen-Tauscher \\
\hline & $4 \% \mathrm{Mg}(\mathrm{OH})_{2}^{b}$ & $\mathrm{PE}+\mathrm{Mg}(\mathrm{OH})_{2}$ & Ionen-Tauscher \\
\hline
\end{tabular}




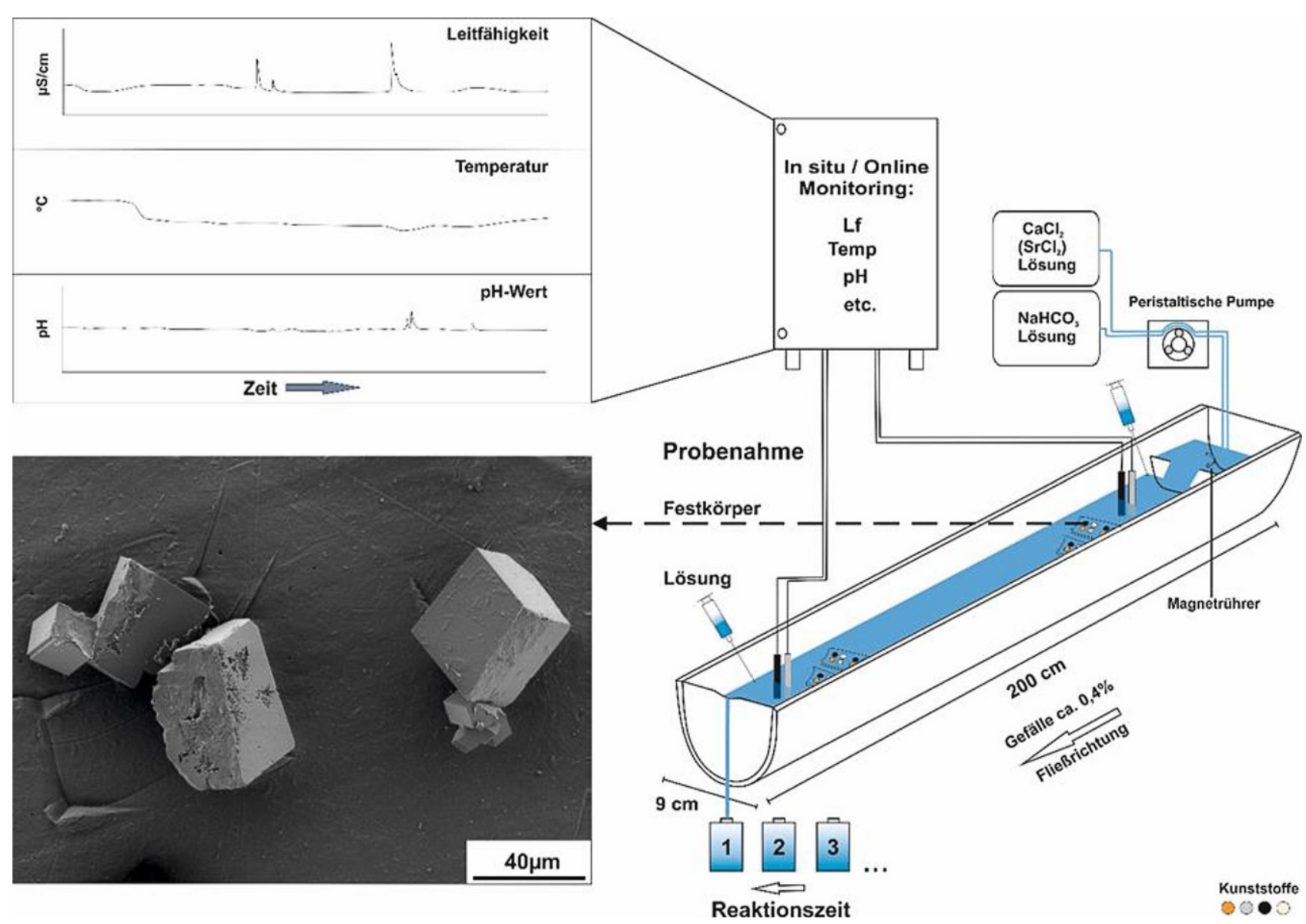

Abb. 1: Versuchsaufbau zur Bestimmung des variablen Versinterungsverhaltens unter dynamischen Bedingungen, inklusive kontinuierlicher Messung von elektrischer Leitfähigkeit, Temperatur und $\mathrm{pH}-$ Wert

Abb. 2: Detailaufnahme des dynamischen Versuchsaufbaus inklusive Darstellung der unterschiedlichen Beobachtungsmöglichkeiten der Sinterablagerung

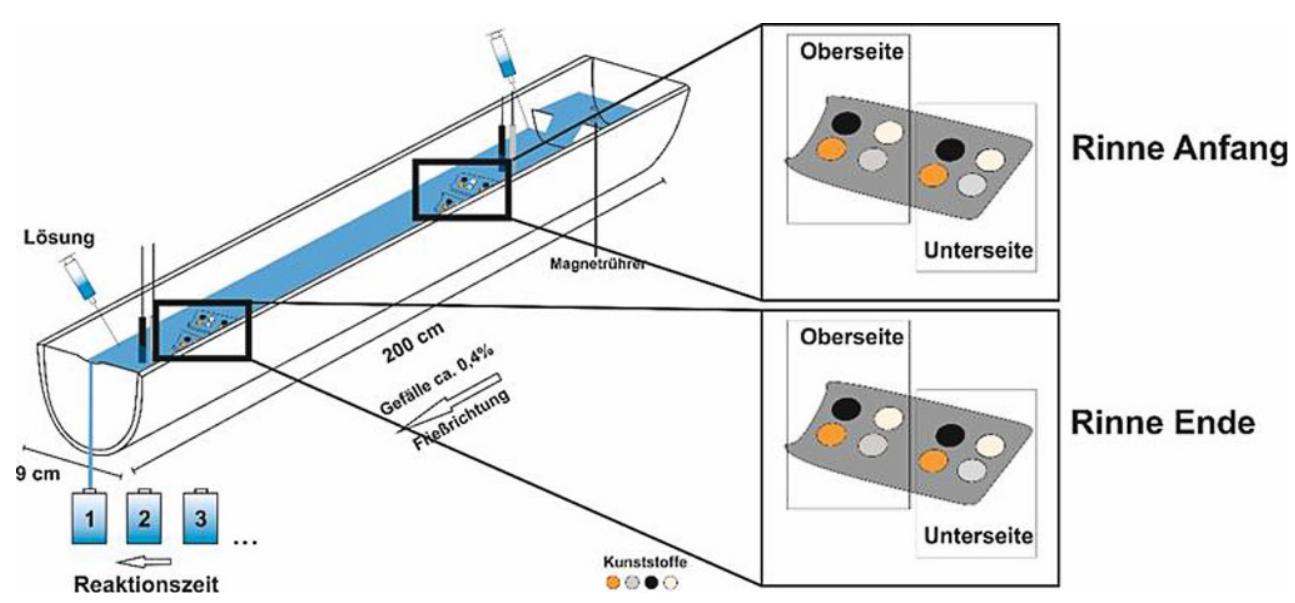

strecken in speziell hierfür angefertigten Halterungen in die Lösung eingebracht worden (Abb. 2). Letztere erlauben eine Differenzierung zwischen Mineral-Aufwachsen und Mineral-Aufwachsen mit Mineral-Sedimentation (heterogene versus homogene Kristall-Nukleation). Während die Abscheidungen an der Oberseite der individuellen Kunststoffe in der Lösung auch durch Mineral-Sedimentation durch in Suspension befindliche Kristalle entstanden sein könn- ten, sind die gebildeten Präzipitate auf der Unterseite der individuellen Kunststoffe ausschließlich an den einzelnen Kunststoffen aufgewachsen. Alle Ergebnisse beziehen sich daher in dieser Arbeit stehts auf die untersuchte Unterseite der Proben.

Im Vergleich zu diesen Laborversuchen unter kontrollierten Randbedingungen wurden zusätzlich Versuche in drei realen Bauwerken durchgeführt. Dazu wurden Proben, wie 

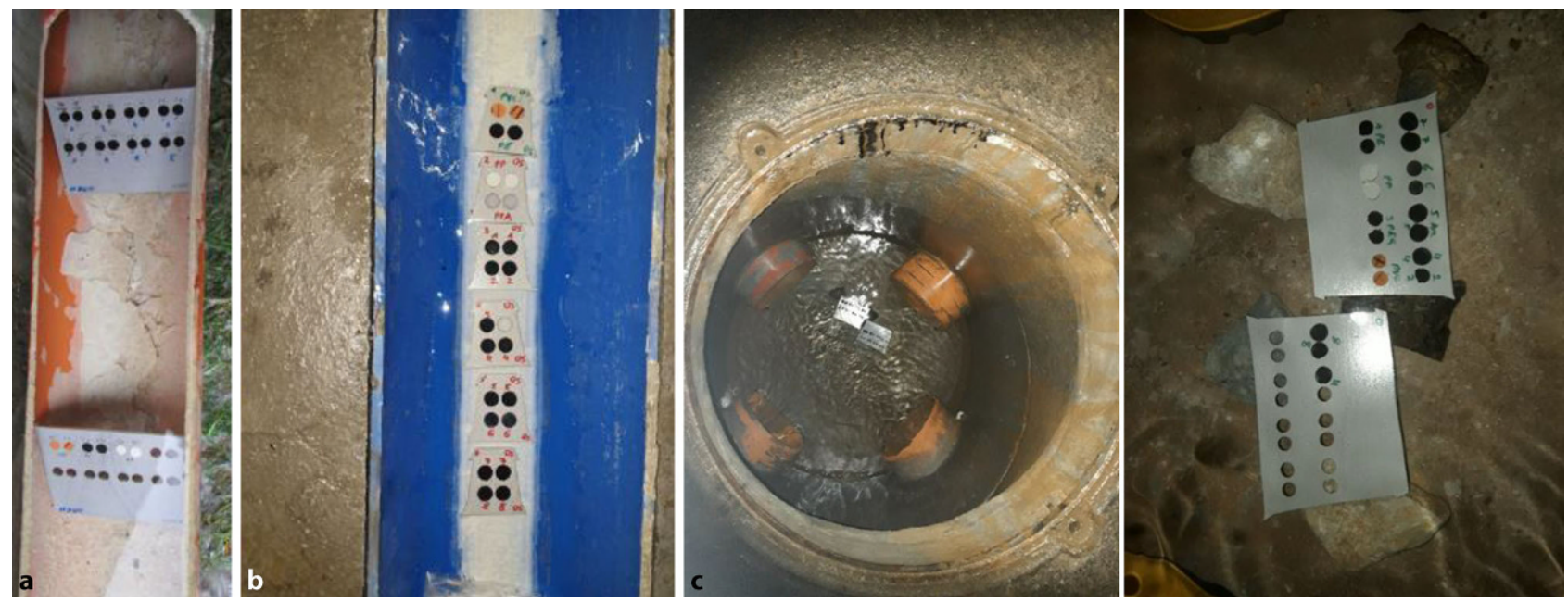

Abb. 3: Auslagerung der Kunststoffproben am Drainageausgang des Tunnels Steinhaus (a), Lainzer Tunnels (b) [1] und Zentrum am Berg (c)

in Abb. 3a-c gezeigt, in den Tunneln Steinhaus (Drainageausgang), Lainzer-Tunnel (Ulmendrainage im Notausstieg Nikolaitor) sowie am Zentrum am Berg (ZaB, Straßentunnel STR Nord und Eisenbahntunnel EBT West) ausgelagert und anschließend untersucht.

\subsubsection{Stationäre Versuche}

Um auch den Einfluss von stehendem und an Kalziumkarbonat übersättigtem Wasser zu untersuchen, wurden in einem zweiten Prüfstand an der TU-Graz ebenfalls Proben (rechteckige Geometrie - $40 \times 25 \mathrm{~mm}^{2}$ für größere Oberflächen zur quantitativen Bewertung) in einem stationären Reaktor ausgelagert (Abb. 4). Der Versuchsaufbau war in der chemischen Zusammensetzung gleich konzipiert wie in den dynamischen Experimenten zuvor, um eine Vergleichbarkeit der einzelnen Versuche zu gewährleisten. Die Versinterungsneigung der Kunststoffe wurde unter stationären Bedingungen (anstelle von dynamischen Bedingungen in der Fließstrecke) untersucht. Unter stationären Bedingungen werden die rechteckigen Kunststoffe mittels Nie-Roster Schrauben befestigt, um das Aufschwimmen in der Wassersäule in einem 1-Liter-Reaktor zu verhindern. Je Reaktor wurden 4 Kunststoffproben exponiert. Ein Magnetrührer mit Rührstäbchen $(400 \mathrm{rpm})$ sorgte für ein moderate aber kontinuierliche Wasserzirkulation im Reaktor (Abb. 4). Die Versuchsdauer betrug 7 Tage.

\subsection{Auswertemethodik \& Beurteilung}

Zur Beurteilung der Wirksamkeit der entwickelten Compounds gegen Versinterung wurden zwei unterschiedliche Aus- bzw. Bewertungsmethoden zu herangezogen. Zum einen wurde eine chemische Analyse mittels Säureaufschluss und zum anderen eine optische Analyse der bedeckten Oberfläche durchgeführt.

Der Säureaufschluss wurde für jeden Kunststoff separat durchgeführt, um über die Kalziumkonzentration die an der jeweiligen Kunststoffoberfläche gebildete Menge der Präzipitate $\left(\mathrm{mg} / \mathrm{cm}^{2}\right)$ zu ermitteln, sowie ein Ranking der Kunststoffe hinsichtlich ihrer Effizienz gegen Versinterung vorzunehmen. Hierfür wurden die während der Auslagerungsversuche gebildeten Kalziumkarbonat-Minerale in $6 \%$ iger $\mathrm{HNO}_{3}$ aufgelöst. Anschließend wurde die Kalziumkonzentration bestimmt (mg/l; mittels ICP-OES Analyse), auf die absolute Menge umgerechnet und der Kunststoff bewertet. Zusätzlich wurden Mikroskopie-basierte Bilder der Kunststoffoberflächen aufgenommen und mittels einer angepassten Bildanalyse-Prozedur untersucht. Bei der Bildanalyse wurden für jeden Kunststoff die kumulierten
Abb. 4: Versuchsreaktoren zur Auslagerung größerer Versuchsproben unter stationären Bedingungen an der TU-Graz
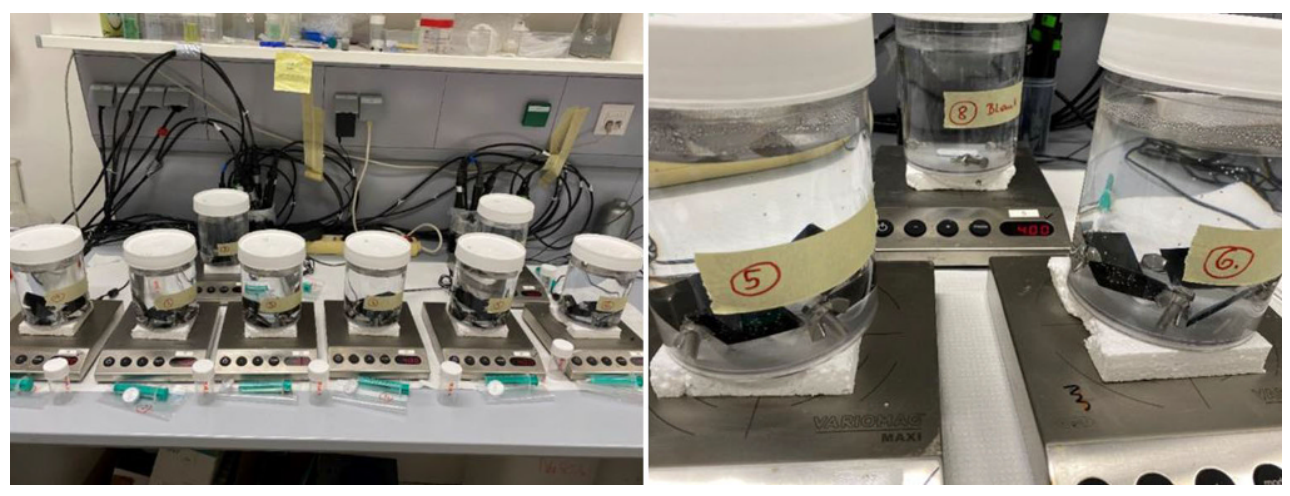
Flächen der Kalzit-Kristallpartikel ins Verhältnis zum untersuchten Bildausschnitt gesetzt.

Zur besseren Vergleichbarkeit der beiden Methoden wurden die Ergebnisse jeweils auf die Ergebnisse des reinen Polyethylens (Matrix) normiert. Dadurch ergibt sich, dass alle Werte unter $100 \%$ eine Reduktion der Versinterungsneigung im Vergleich zu Polyethylen und Werte $>100 \%$ eine Erhöhung der Versinterungsneigung des jeweiligen Kunststoffs bedeuten. In den Ergebnis-Graphen wurde dies zusätzlich noch farbig herausgestellt, mit einer grünen Färbung für eine Reduktion, und roter Färbung für eine Erhöhung der Versinterungsneigung im Vergleich zum reinen Matrixwerkstoff.

\section{Ergebnisse}

\subsection{Auswertemethodik \& Beurteilung}

Zur quantitativen Auswertung der Versinterungsneigung wurden die Methode des Säureaufschlusses sowie eine optische Bewertung durchgeführt. Als erster Schritt sollte die Vergleichbarkeit beider Methoden festgestellt werden. Dazu wurden Proben aus verschiedenen Auslagerungsorten mit beiden Methoden bewertet und verglichen. Die Ergebnisse dazu sind exemplarisch für den dynamischen Versuch in der Rinne, bzw. für die Auslagerung im Lainzer-Tunnel in Abb. 5 dargestellt. Wie in beiden Ergebnissen ersichtlich ist, zeigen beide Methoden vergleichbare Resultate. Während die exakten Einzelbewertungen nicht immer exakt übereinstimmen, so zeigen beide Methoden jedoch mit relativ hoher Zuverlässigkeit die gleichen Trends und GröBenordnungen an.

\subsection{Auslagerungsversuche}

Nachfolgend sind die Ergebnisse der durchgeführten Auslagerungsversuche dargestellt. Die Ergebnisse beinhalten sowohl die dynamischen Versuche (sowohl Labor- als auch Feldversuch), als auch die stationären Versuche im Reaktor. Zusätzlich werden auch die Ergebnisse der wiederholten Auslagerungen (dynamisch am ZaB, bzw. stationär im Reaktor) gezeigt. Diese Untersuchungen dienen primär dazu festzustellen, ob die gewünschten Effekte auch nach mehrmaliger Reinigung noch auftreten.

\subsubsection{Dynamische Versuche}

Bei der Durchführung der ersten dynamischen Versuche im Laborprüfstand der TU-Graz wurden 6 verschiedene Compounds untersucht, da der Wirkstoff $\mathrm{Mg}(\mathrm{OH})_{2}$ erst im späteren Projektverlauf mitaufgenommen wurde. Sowohl in der optischen Analyse als auch im Säureaufschluss wurden die besten Ergebnisse (wenig Versinterung) mit den Compounds bestehend aus PE + PEG, PE + Zeolith, $\mathrm{PE}+$ Ammoniumphosphat und $\mathrm{PE}+\mathrm{Na}-\mathrm{Stearat}$ erzielt (vgl. Abb. 5). Zusätzliche Analysen mittels Rasterelektronenmikroskop (REM) zeigten schön (idiomorph) ausgebildete Kalzitkristalle auf allen untersuchten Proben (siehe beispielhaft Abb. 1).

Ebenfalls in Abb. 5 dargestellt sind die Ergebnisse der Proben aus dem Lainzer-Tunnel. Abermals fanden sich vor allem PE + PEG, PE + Zeolith, PE + Ammoniumphosphat und $\mathrm{PE}+\mathrm{Na}$-Stearat unter den besten der untersuchten Wirkstoffe. Die Proben, die am Drainageausgang des Tunnels Steinhaus platziert waren, wurden bei einer Tunnelspülung leider beschädigt und konnten nicht ausgewertet werden.

Die letzten Proben in dynamischen Systemen wurden am Zentrum am Berg in Eisenerz ausgelagert. Die Proben wurden dabei in Schächte des Straßentunnels STR Nord bzw. des Eisenbahntunnels EBT West eingebracht und für eine bzw. drei Wochen ausgelagert. Bemerkenswert ist dabei der Unterschied bzw. generell die Höhe der pHWerte der Gewässer (Nord; $\mathrm{pH}=11,5$ und Süd $\mathrm{pH}=8,5$ ). Bei einem sehr hohen $\mathrm{pH}$-Wert von 11,5 entstanden auf den ausgelagerten Versuchsproben bereits nach einer Woche starke Versinterungen. Interessanterweise schaffte
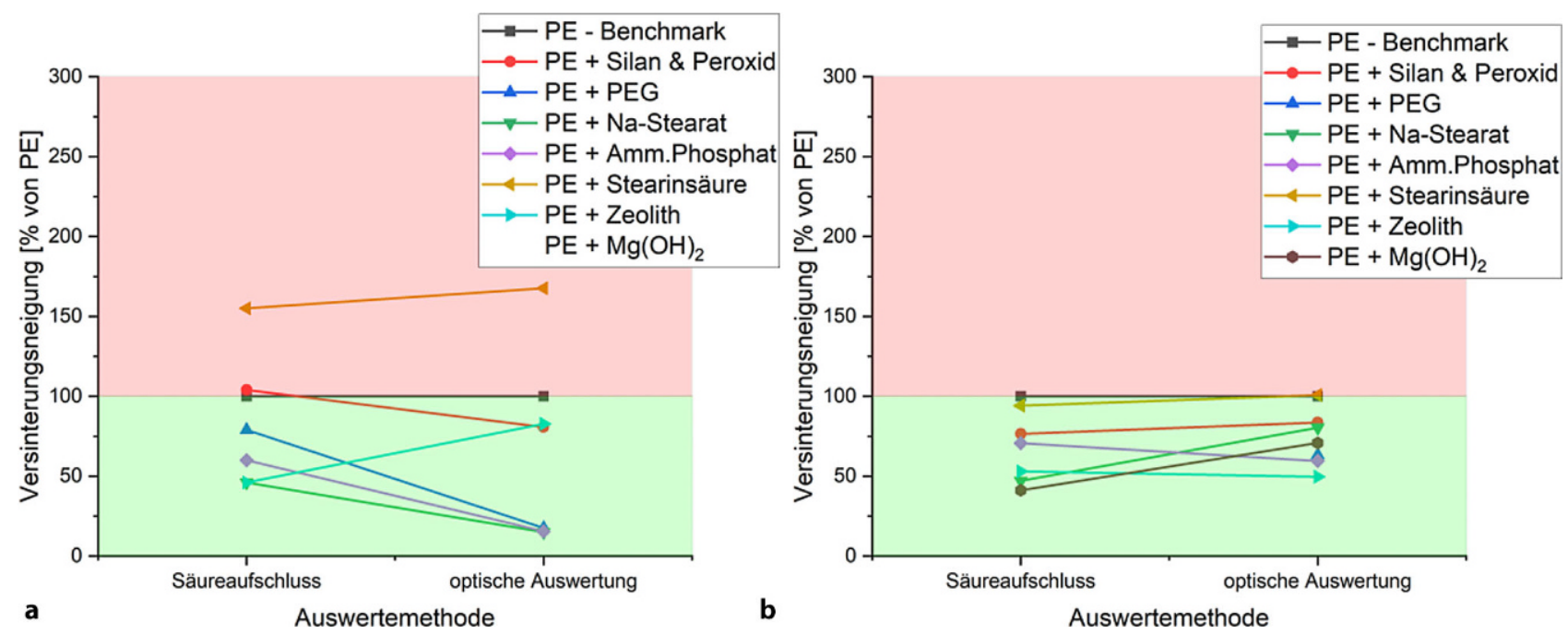

Abb. 5: Vergleich der Auswertemethodik am Beispiel der dynamischen Versuche an der TU-Graz, bzw. der ausgelagerten Plättchen im Lainzer-Tunnel 


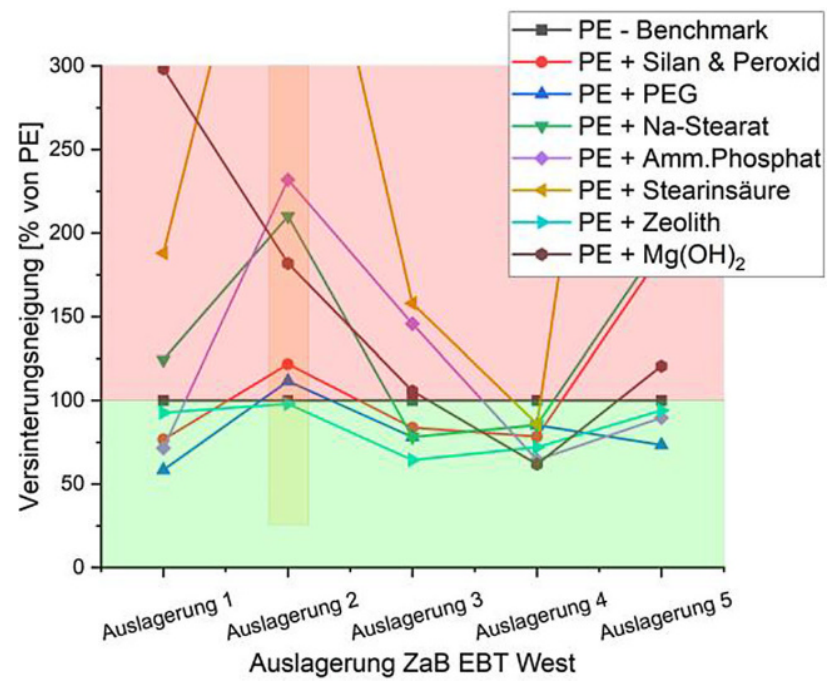

Abb. 6: Ergebnis der wiederholten Auslagerung am ZaB EBTWest es hier das nicht modifizierte Matrixmaterial (PE) in die Top 3 des Rankings (Abb. 8). Dies könnte eine Indikation dafür sein, dass bei derart hohen pH-Werten die gewählten Modifikationen im Vergleich zu moderateren Milieus nicht wirksam sind. Bei den Proben, die an der Kreuzung Süd und einem gemäßigten $\mathrm{pH}$-Wert von $~ 8,5$ ausgelagert wurden, zeigte sich hingegen primär wieder die gewohnte Reihenfolge.

Um zu überprüfen, ob die aufgetretenen Effekte der untersuchten Compounds nur einmalig oder auch mehrmals wirksam sind, wurden dieselben Proben mehrmals in der Auslagerungsstelle ZaB EBT West ausgelagert, ausgewertet und gereinigt. Dieser Vorgang wurde, wie in Abb. 6 gezeigt, in Summe fünfmal wiederholt. Mit Ausnahme der zweiten Messung, bei der die Werte nach oben versetzt sind, zeigten die Messungen 1, 3, 4 und 5 ähnliche Trends und vor allem die Compounds mit PEG und Zeolith zeigten konstant gute Ergebnisse. Die Analyseergebnisse der zweiten Messung lassen sich durch die Einleitung von Bergwasser über zusätzliche Rohrleitungen erklären, wofür der

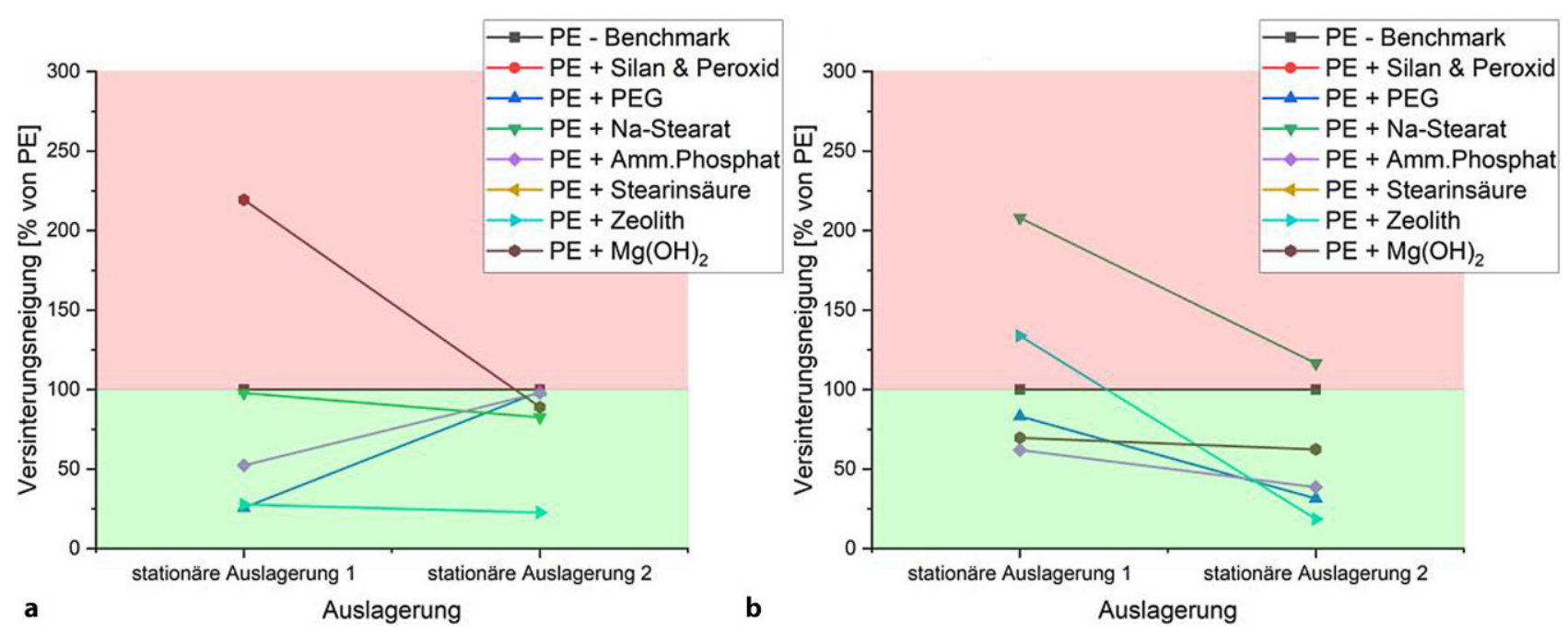

Abb. 7: Ergebnis der wiederholten Auslagerung im stationären Versuchsreaktor an der TU-Graz-Auswertung mittels Säureaufschluss und optischer Analyse der Oberfläche

Abb. 8: Zusammenfassung und Wertung aller Untersuchungen hinsichtlich der Versinterungsneigung der verwendeten PE-Kunststoffe mit Zusatzstoffen

\begin{tabular}{|c|c|c|c|c|c|}
\hline $\begin{array}{c}\text { Bauwerk / } \\
\text { Bezeichnung }\end{array}$ & $\begin{array}{l}\text { Auswerte- } \\
\text { methode }\end{array}$ & 1. Platz & 2. Platz & 3. Platz & $\mathrm{pH}$ \\
\hline \multicolumn{6}{|c|}{ dynamische Versuche } \\
\hline \multirow{2}{*}{ Rinne } & Optisch & PE+Amm.Phosphat & $\mathrm{PE}+\mathrm{Na}$-Stearat & PE+PEG & \multirow{2}{*}{ 7.8-8.1 } \\
\hline & Säureaufschluss & PE+Zeolith & $\mathrm{PE}+\mathrm{Na}$-Stearat & PE+Amm.Phosphat & \\
\hline \multirow{2}{*}{ Lainzer Tunnel } & Optisch & PE+Zeolith & PE+Amm.Phosphat & PE+PEG & \multirow{2}{*}{ 8.1-8.5 } \\
\hline & Säureaufschluss & $\mathrm{PE}+\mathrm{Mg}(\mathrm{OH}) 2$ & $\mathrm{PE}+\mathrm{Na}$-Stearat & PE+Zeolith & \\
\hline \multirow{2}{*}{ ZaB Süd } & Optisch & PE+Zeolith & PE+PEG & PE+Silan & \multirow{2}{*}{8.5} \\
\hline & Säureaufschluss & PE+Zeolith & PE+Amm.Phosphat & PE & \\
\hline \multirow{2}{*}{ ZaB Nord } & Optisch & $\mathrm{PE}+\mathrm{PEG}$ & PE & PE+Silan & \multirow{2}{*}{11.5} \\
\hline & Säureaufschluss & PE & PE+Zeolith & $\mathrm{PE}+\mathrm{Na}$-Stearat & \\
\hline \multicolumn{6}{|c|}{ statische Versuche } \\
\hline \multirow{2}{*}{ Statischer Versuch 1} & Optisch & PE+Amm.Phosphat & $\mathrm{PE}+\mathrm{Mg}(\mathrm{OH}) 2$ & $\mathrm{PE}+\mathrm{PEG}$ & \multirow{4}{*}{ 7.7-8.1 } \\
\hline & Säureaufschluss & PE+PEG & PE+Zeolith & PE+Amm.Phosphat & \\
\hline \multirow{2}{*}{ Statischer Versuch 2} & Optisch & PE+Zeolith & PE+PEG & PE+Amm. Phosphat & \\
\hline & Säureaufschluss & PE+Zeolith & $\mathrm{PE}+\mathrm{Na}$-Stearat & $\mathrm{PE}+\mathrm{Mg}(\mathrm{OH}) 2$ & \\
\hline
\end{tabular}




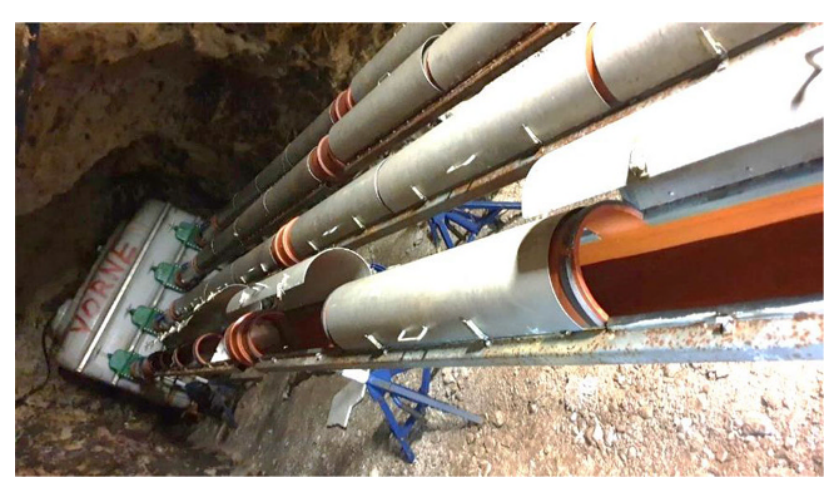

Abb. 9: Geplante Versuchsstrecke für Drainagerohre, hergestellt aus den modifizierten Kunststoffen am ZaB

Schacht für Rohre angebohrt werden musste und von einer kurzzeitig erhöhten Mobilisierung von Stoffen ausgegangen werden kann, die die Versinterungsneigung steigerte. Darauf kann auch der abfallende Trend der Versinterungsneigung bei den Auslagerungen 2 bis 4 gründen.

\subsubsection{Stationäre Versuche}

Wie eingangs erwähnt, wurden neben den dynamischen auch stationäre Versuche durchgeführt. Der primäre Unterschied dabei ist, dass die angesetzten Lösungen im Reaktor nicht wie bei fließenden Gewässern ausgetauscht (erneuert) werden. Analog zu den Wiederholungsmessungen im ZaB EBT West wurden die Proben dieser Versuche ebenfalls nach den ersten Untersuchungen gereinigt und abermals ausgelagert. Die Ergebnisse zu den stationären Versuchen sind nachfolgend in Abb. 7 dargestellt. An dieser Stelle sei angemerkt, dass die Compounds mit Silan und Stearinsäure in diesen zusätzlichen Versuchen, basierend auf den wenig aussichtsreichen Ergebnissen der vorangehenden Untersuchungen, nicht weiter untersucht wurden.

Wie ersichtlich, sind auch im stationären Versuch vor allem wieder PE mit PEG, Zeolith und Polyphosphat unter den besonders effizienten Materialien vertreten.

\subsubsection{Zusammenfassung der Ergebnisse}

Zur übersichtlichen Darstellung wurden die drei hinsichtlich reduzierter Versinterungsneigung jeweils aussichtsreichsten Kunststoff-Compounds aller Auslagerungsversuche nachfolgend in Abb. 8 zusammengefasst. Durch diese Form der Darstellung ist es möglich, relativ schnell grundsätzliche Aussagen über die generelle Wirksamkeit der Compounds zu treffen. Die Farben dienen nur zur vereinfachten Wahrnehmung der jeweils gleichen Compounds, haben aber keine Bedeutung hinsichtlich der Wirksamkeit o. ä. Ausgehend von dieser Tabelle und den Platzierungen, scheint das PE-Compound mit Zeolith unter den gegebenen Auslagerungsbedingungen am besten gegen Versinterung zu wirken. Auf dem zweiten Platz, mit annähernd gleicher Effizienz, folgen die Compounds mit Polyethylen-Glykol, sowie Ammonium-Polyphosphat.

\section{Zusammenfassung}

Basierend auf den in dieser Studie erhaltenen Ergebnissen scheinen die Kunststoff-Compounds mit Zeolith, Polyethylen-Glykol und Ammoniumphosphat das Versinterungsverhalten positiv, im Sinne einer geringeren Neigung zur Sinterbildung, zu beeinflussen. Bei sehr hohen pH-Werten (>>9) führen jedoch extrem hohe Übersättigungen an $\mathrm{CaCO}_{3}$ Mineralen zu sehr raschen Versinterungen auf den exponierten Kunststoff-Oberflächen, was die Effizienz der Wirkstoffe deutlich begrenzen kann. Daher sollten die gefundenen Ergebnisse in Bauwerken mit sehr hohen $\mathrm{pH}$ Werten mit Vorsicht betrachtet werden. Nichtsdestoweniger ist als logischer nächster Schritt ein "upscaling“ der gefundenen Compounds auf reale Rohrgeometrien, mit anschließender Versuchsreihe in Bauwerken, anzusetzen. Dazu wird derzeit mit Rohrherstellern an einer Umsetzung gearbeitet. Als erster Aufstellungsort für Versuche mit realen Rohrgeometrien wäre der in Abb. 9 gezeigte Aufbau am Zentrum am Berg geplant. Dabei können vier Rohrstrecken DN200 mit realem Bergwasser gespeist und hinsichtlich der Versinterungsneigung untersucht werden.

Danksagung. Die durchgeführten Arbeiten in dieser Veröffentlichung wurden im Rahmen des VIF-2016 Projekts „PolyDrain“ (860529) mit Beteiligung der ÖBBInfrastruktur AG, sowie der ASFINAG und des Bundesministeriums für Verkehr, Innovation und Technologie durchgeführt.

Funding. Open access funding provided by Montanuniversität Leoben.

Open Access Dieser Artikel wird unter der Creative Commons Namensnennung 4.0 International Lizenz veröffentlicht, welche die Nutzung, Vervielfältigung, Bearbeitung, Verbreitung und Wiedergabe in jeglichem Medium und Format erlaubt, sofern Sie den/die ursprünglichen Autor(en) und die Quelle ordnungsgemäß nennen, einen Link zur Creative Commons Lizenz beifügen und angeben, ob Änderungen vorgenommen wurden.

Die in diesem Artikel enthaltenen Bilder und sonstiges Drittmaterial unterliegen ebenfalls der genannten Creative Commons Lizenz, sofern sich aus der Abbildungslegendenichts anderes ergibt. Sofern das betreffende Material nicht unter der genannten Creative Commons Lizenz steht und die betreffende Handlung nicht nach gesetzlichen Vorschriften erlaubt ist, ist für die oben aufgeführten Weiterverwendungen des Materials die Einwilligung des jeweiligen Rechteinhabers einzuholen.

Weitere Details zur Lizenz entnehmen Sie bitte der Lizenzinformation auf http://creativecommons.org/licenses/by/4.0/deed.de.

\section{Literatur}

1. Arbeiter, F.; Eichinger, S.; Rieß, G.; Schachinger, T.; Boch, R.; Wenighofer, R.; Galler, R.; Hausberger, A.; Strobl, E.; Stur, M.; Saliger, F; Steiner, M.; Dietzel, M., Pinter; G.: Optimierte Polymer-Rohrwerkstoffe für effiziente Drainagesysteme in Tunnelbauwerken - PolyDrain. Berg- u. Hüttenmännische Monatshefte 164 (2019), H. 12, S. 545-551. https://doi.org/10.1007/s00501-019-00918-6

2. Schachinger, T.; Zagar, B.; Schwab, C.; Saliger, F; Stur, M.: Current research by ÖBB Infrastruktur AG on scale monitoring without track closures. Geomechanik Tunnelbau 11 (2018), iss. 3, pp 277-285. https://doi.org/10.1002/geot.201800012

3. Schachinger, T.; Arbeiter, F.J.; Eichinger, S.; Saliger, F.: Research on pipe materials for tunnel drainage by the ÖBB Task Force Drainage. Geomechanik und Tunnelbau 12 (2019), iss. 5, pp 467-471. https:// doi.org/10.1002/geot.201900022 
4. Siegmann, K.; Sterchi, R.; Widler, R.; Hirayama, M.: Lime repellent polyethylene additives. Journal of Appied Polymer Science 129 (2013), iss. 5, 2727-2734. https://doi.org/10.1002/app.38928.

5. Dietzel, M.; Rinder, T.; Niedermayr, A.; Mittermayr, F.; Leis, A.; Klammer, D.; Köhler, S.; Reichl, P.: Ursachen und Mechanismen der Versinterung von Tunneldrainagen. Berg- und Hüttenmännische Monatshefte 153 (2008), H. 10, S. 369-372. https://doi.org/10.1007/ s00501-008-0412-3

6. Dietzel, M.; Kieffer, D.S.; Schubert, W.; Schweiger, H.; Semprich, S.: Drainagesysteme im Tunnelbau: Design, Versinterung und Instandhaltung. Graz: Verlag der Technischen Universität Graz; 2008

7. Eichinger S, Leis A, Boch R, Seywald C, Dietzel M. Assessment and formation mechanisms of scale deposits in tunnels of the ÖBB-Infrastruktur AG-A subproject of the Task Force Drainage // Bewertung von Sinter und deren Bildungsbedingungen in Tunnelbauwerken der ÖBB-Infrastruktur AG - Ein Teilprojekt der Task
Force Drainage. Geomechanik Tunnelbau 2020;13(3):273-85. https:// doi.org/10.1002/geot.202000006.

8. Eichinger, S.; Boch, R.; Leis, A.; Koraimann, G.; Grengg, C.; Domberger, G.; Nachtnebel, M.; Schwab, C.; Dietzel, M.: Scale deposits in tunnel drainage systems-A study on fabrics and formation mechanisms. Science of the total environment 718 (2020),137140. https:// doi.org/10.1016/j.scitotenv.2020.137140.

9. Eichinger, S.; Arbeiter, F.J.; Boch, R.; Schachinger, T.; Dietzel, M.: Influencing Carbonate Scaling by Tailored Drainage Materials in Tunnels-An Experimental Approach. In: Austrian Mineralogical Society, editor. MinPet 2019. Graz: Verein Österreichische Mineralogische Gesellschaft; 2019, p. 34

Hinweis des Verlags. Der Verlag bleibt in Hinblick auf geografische Zuordnungen und Gebietsbezeichnungen in veröffentlichten Karten und Institutsadressen neutral. 